\title{
COMPARISON OF SUBJECTIVE AND OBJECTIVE EVALUATION OF SCREEN-FILM SYSTEMS FOR CHEST RADIOGRAPHY
}

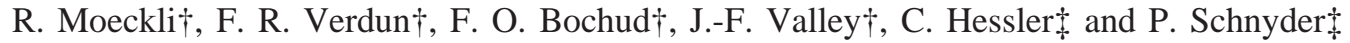 \\ $\dagger$ Institute of Applied Radiophysics, University Centre \\ CH-1015 Lausanne, Switzerland \\ †University Hospital Centre (CHUV), Department of Diagnostic and Interventional Radiology \\ CH-1011 Lausanne, Switzerland
}

\begin{abstract}
A subjective and an objective comparison of six screen-film systems is reported. Among the objective parameters which characterise image quality, resolution appeared to be the most critical one when compared with the averaged ranking produced by the radiologists. The results have shown that a relationship between dose and image quality can be established for most of screen-film systems tested. The problem which remains in the optimisation procedure of chest imaging, is the definition of the level of image quality requirements.
\end{abstract}

\section{INTRODUCTION}

In the field chest radiography as in other fields, new screen-film systems are regularly appearing on the market. In order to be able to make an optimal choice concerning patient dose and image quality, one has to evaluate the performance of these new materials or systems and compare the results. In radiology the most obvious way to make comparisons is to try the new materials on different patients, or better on an anthropomorphic phantom, to avoid anatomy variations. The problem with this methodology is that, in most cases, the differences to be observed are so small that the results of the comparison are inconclusive. The ideal way to evaluate new systems would be to use fully objective tests leading to a single number. Optimisation of radiology would consist then in choosing the most sensitive system which only carries the required clinical information. The problem with this concept is that the required clinical information varies from patient to patient. Thus, complete optimisation, that is a tailored optimisation scheme, is utopian. But at the present time, even an optimisation scheme based on a simple balance between patient dose and the standard amount of clinical information is impossible to set up.

The goal of the study is to compare objectively and subjectively six recent screen-film systems in order to verify whether a correlation between these two methodologies can be found. The relationship between dose and image quality is also evaluated.

\section{MATERIAL AND METHOD}

All the measurements have been performed using a Toshiba standard radiographic installation (KXO-50R) which has a high frequency generator. High voltage values produced by the generator were controlled by means of a conventional digital kilovoltmeter (Victoreen 4000+, Nuclear Associates, USA). The measured doses were corrected according to calibration factors determined using a secondary standard consisting of a NE 2575 ionisation chamber and a NE 2560 electrometer, whose calibration is traceable to the primary standard of the National Physical Laboratory (NPL, UK). The sensitivity of the screen-film systems was measured according to the 9236 ISO standard using the fourth beam quality (i.e. high voltage of $120 \mathrm{kV}$; HVL of $8.5 \mathrm{~mm}$ equivalent aluminium $)^{(1)}$. Film processing was controlled according to DIN 6868 Part 55 standard $^{(2)}$, so film processing was always in agreement with the manufacturer's recommendations. The screen-film systems involved in the study are reported in Table 1.

\section{Subjective assessment}

A radiograph of a standard anthropomorphic chest phantom was taken with each system on the unit previously mentioned. Each radiograph was performed at $125 \mathrm{kV}$, with a focus-to-film distance of $1.8 \mathrm{~m}$, adjusting the tube loading in order to obtain an optical density of $1.5 \pm 0.1$ in the lung area. The films were ranked by five experienced radiologists, according to their own criteria. Two rankings were produced: one concerning the image quality in the lung area, and another one in the mediastinal area.

Table 1. Description of the screen-film systems involved in the study.

\begin{tabular}{|c|c|c|c|}
\hline Manufacturer & Screen & Film & Asymmetry properties \\
\hline Kodak & $\mathrm{VHC}$ & ITC-1 & $\begin{array}{l}\text { Thickness of screens } \\
\text { and emulsions }\end{array}$ \\
\hline Dupont & UV-Rapid & UV-G & None \\
\hline Fuji & $\mathrm{AD}$ & UR-1 & Emulsions \\
\hline Typon & OG 800 & TXR-OL & None \\
\hline Agfa & Opthos D & HT-C & Thickness of screens \\
\hline Imation & Chest & GCA & $\begin{array}{l}\text { Screen emissions, film } \\
\text { spectral sensitivity }\end{array}$ \\
\hline
\end{tabular}




\section{R. MOECKLI, F. R. VERDUN, F. O. BOCHUD, J.-F. VALLEY, C. HESSLER and P. SCHNYDER}

\section{Objective assessment}

In order to characterise the different systems in a realistic way, the basic parameters of image quality (i.e. contrast, noise and resolution) were measured using a test object which simulates the $\mathrm{X}$ ray absorption of an average adult. This test object is a modified version of the test object recommended by the AAPM for patient dose assessment in the field of chest radiography ${ }^{(3)}$. To simulate the mediastinal area, and thus include the dynamic range in the assessment, a $1 \mathrm{~cm}$ thick central slab of aluminium was added in the middle of the test object. Two devices containing step-wedges of PMMA (Plexiglas) in air, aluminium, Teflon and a resolution test pattern have been placed in the test object (see
Figure 1). This test object has been imaged in the same conditions as the anthropomorphic phantom.

To characterise the image quality objectively, all the films have been digitised using a pixel size of $20 \mu \mathrm{m}$ and a dynamic range of 14 bits by means of a Linotype-Hell Tango scanner (Linotype-Hell, Kiel, Germany). The data produced by the scanner have been converted in diffuse optical density by means of a calibrated step-wedge. The contrast, noise and resolution measurements of the films were performed at two optical density levels in order to evaluate the systems in the lung and mediastinal region. Low and high contrast Teflon-PMMA and aluminiumPMMA were measured by means of the step-wedges. The resolution was assessed according to the methodology described in the DIN 6867 Part $2^{(4)}$. The MTF was calcu-
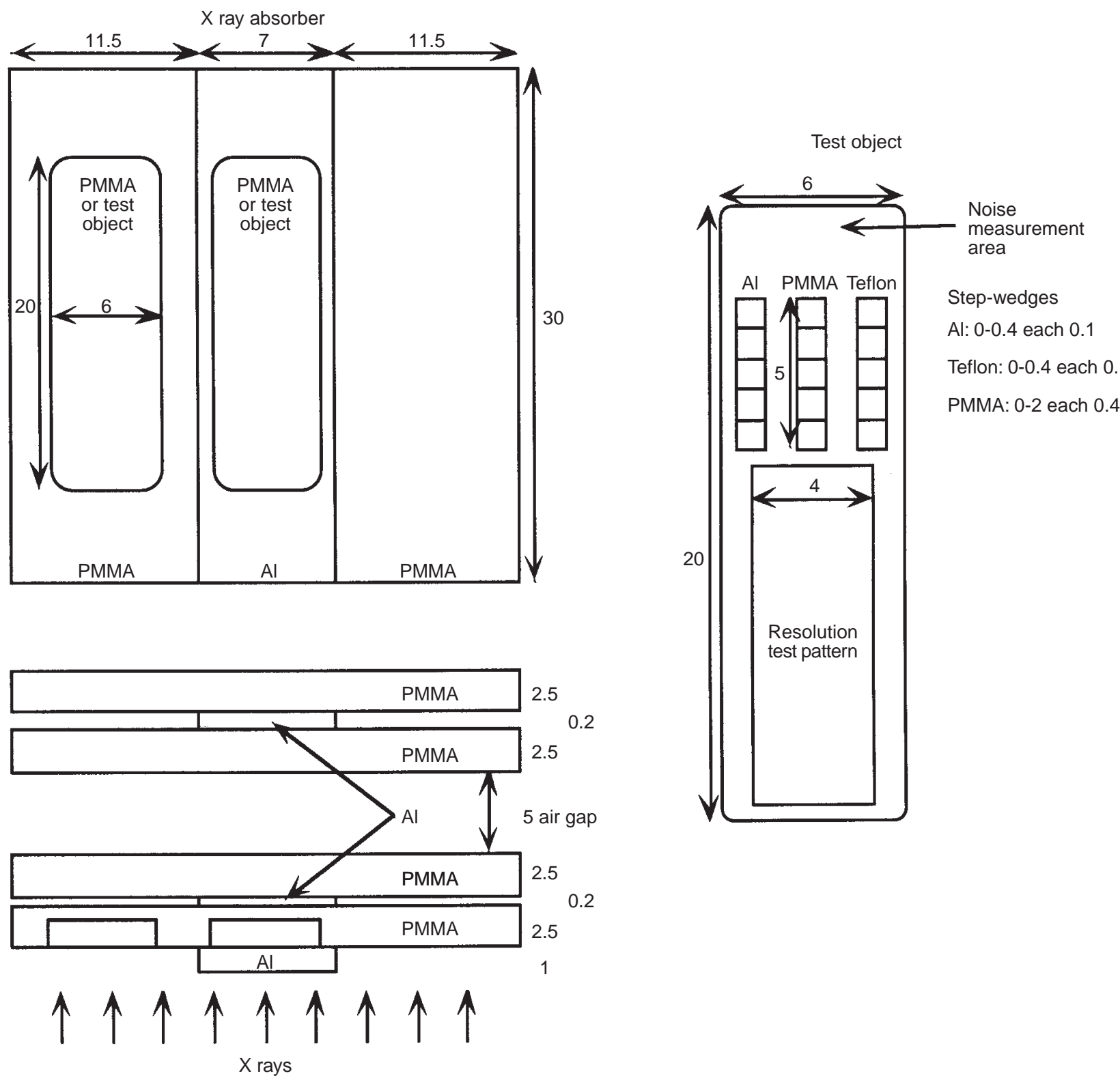

Figure 1. Schematic diagram of the test object used in the study. All measurements are in $\mathrm{cm}$. 

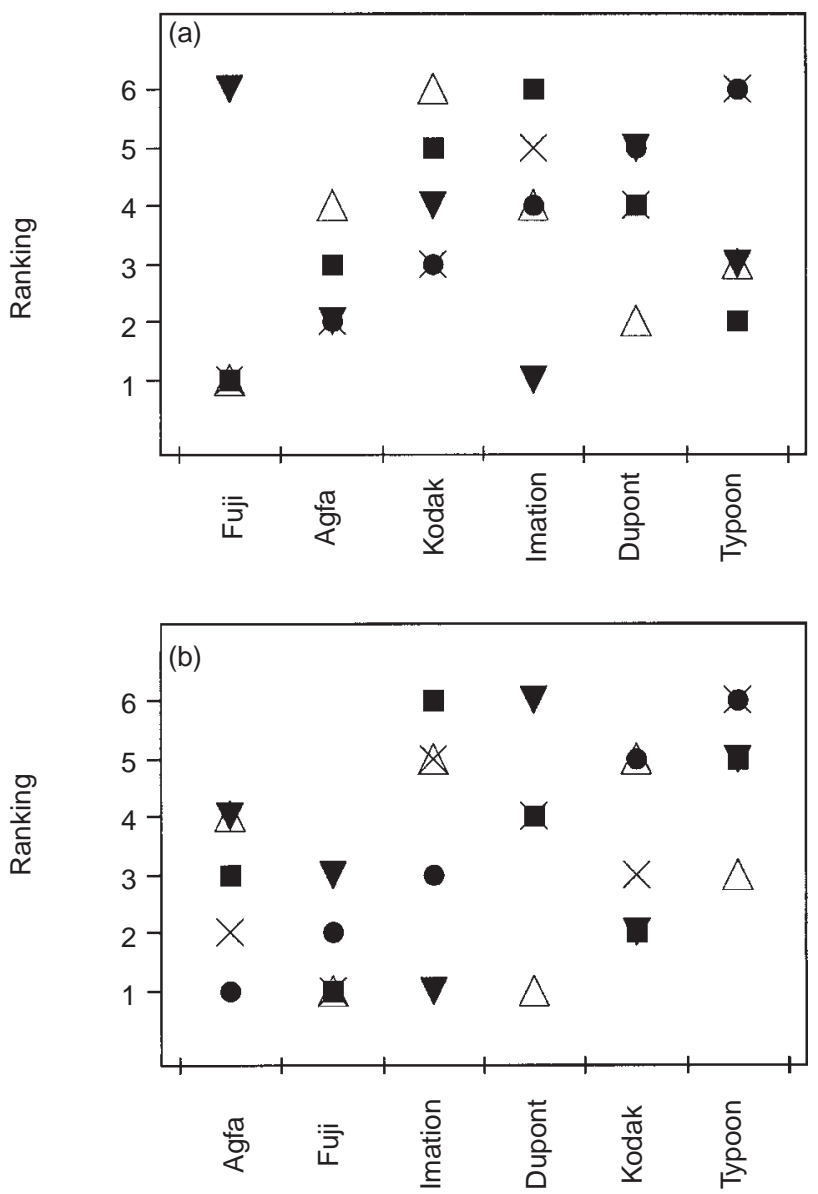

Figure 2. Comparison of the averaged ranking of the radiologists with the contrast, noise, resolution and GFM rankings. (a) The results obtained in the lung area. (b) The results obtained in the mediastinal area. $(\triangle)$ Contrast, $(\boldsymbol{\nabla})$ noise, $(\times)$

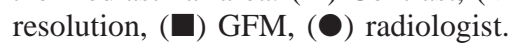

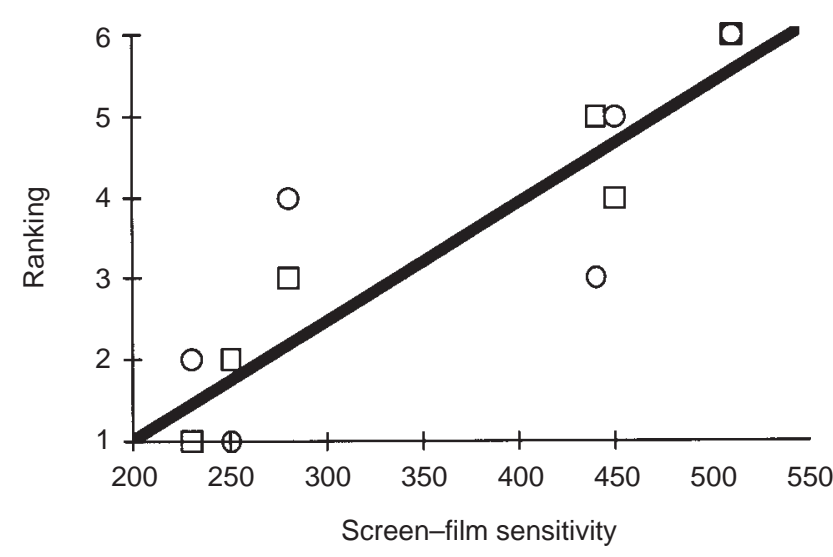

Figure 3. Relationship between averaged ranking of the radiologists in the lung $(\bigcirc)$ and mediastinal $(\square)$ areas and the sensitivity of the system. lated using the methods described by Coltman ${ }^{(5)}$. The Wiener noise power spectrum was obtained by performing a $2 \mathrm{D}$ FFT on four $256 \times 256$ data sets. The averaging of these spectra was followed by a polar averaging in order to get a one dimensional spectrum.

In order to characterise the different systems by a unique quantity, a global figure of merit (GFM), based on the evaluation of the integral of the signal-to-noise ratio of an ideal observer ${ }^{(6)}$ detecting a high contrast punctual object was calculated. This quantity was evaluated by means of the following equation:

$$
\mathrm{GFM}=\mathrm{C}^{2} \int_{0}^{10} \frac{\mathrm{MTF}^{2}(\mathrm{f}) \mathrm{df}}{\mathrm{WS}(\mathrm{f})}
$$

where $\mathrm{C}$ is the contrast aluminium expressed in optical density per mm of aluminium, MTF and WS are the modulation transfer function and the Wiener noise power spectrum respectively. These parameters were also measured with the test object presented in Figure 1.

\section{RESULTS AND DISCUSSION}

The results of the rankings of the individual radiologists for the lung and mediastinal regions are reported in Tables 2 and 3. In these tables, the lowest number was given to the best system. An averaged ranking, based on the sum of all the grades given by the radiologists, have also be introduced. From these results a mean rank was deduced. It is interesting to note that there is a better agreement among the radiologists for

Table 2. Rankings of the radiologists for the lung area (optical density between 1.43 and 1.61).

System Radiol. Radiol. Radiol. Radiol. Radiol. Average

\begin{tabular}{lllllll} 
& 1 & 2 & 3 & 4 & 5 & ranking \\
\hline Kodak & 4 & 2 & 1 & 5 & 2 & 3 \\
Dupont & 5 & 5 & 4 & 2 & 3 & 5 \\
Fuji & 1 & 4 & 3 & 1 & 1 & 1 \\
Typon & 6 & 6 & 6 & 4 & 5 & 6 \\
Agfa & 2 & 3 & 1 & 3 & 4 & 2 \\
Imation & 3 & 1 & 5 & 5 & 5 & 4 \\
\hline
\end{tabular}

Table 3. Rankings of the radiologists for the mediastinal area (optical density between 0.78 and 0.93 )

System Radiol. Radiol. Radiol. Radiol. Radiol. Average

\begin{tabular}{lllllll} 
& 1 & 2 & 3 & 4 & 5 & ranking \\
\hline Kodak & 5 & 5 & 4 & 5 & 4 & 5 \\
Dupont & 4 & 4 & 3 & 2 & 3 & 4 \\
Fuji & 3 & 1 & 5 & 2 & 2 & 2 \\
Typon & 6 & 6 & 6 & 5 & 6 & 6 \\
Agfa & 1 & 2 & 1 & 1 & 1 & 1 \\
Imation & 2 & 3 & 2 & 4 & 5 & 3 \\
\hline
\end{tabular}




\section{R. MOECKLI, F. R. VERDUN, F. O. BOCHUD, J.-F. VALLEY, C. HESSLER and P. SCHNYDER}

the best and worst system in the mediastinal region than in the lung region.

Table 4 reports the results obtained with the objective methodology. The contrast values were obtained on the digitised images. They are expressed in optical density per millimetre of aluminium or Teflon. The resolution is given for a spatial frequency of $3 \mathrm{lp} \cdot \mathrm{mm}^{-1}$, and the noise is characterised by the amplitude of the Wiener spectrum at frequency equal to $0.51 \mathrm{p} \cdot \mathrm{mm}^{-1}$. No effect of the optical density level on resolution was noticed ${ }^{(7)}$, thus Table 4 only reports one value for this particular parameter. In order to check if a relationship existed between the ranking of the radiologists and the measurements presented in Table 4, each parameter was ranked and compared with the average rankings produced by the radiologists. In the ranking procedure of the image parameters, the lowest number was given to the highest contrast, highest resolution, highest GFM and lowest noise level. The results are presented in Figure 2.

A very good correlation between the averaged ranking produced by the radiologists and the resolution parameter is demonstrated in the lung area. This correlation is weaker in the case of the mediastinal area, but still exists. No other obvious correlation appears in Figure 2. These results clearly show that the radiologists have put an important weight on resolution when they were asked to assess image quality. This result was surprising to us, since noise is generally the parameter which is mentioned when asking radiologists to compare slow and fast screen-film systems.
In Figure 3 the radiologists' rankings have been represented as a function of the systems' sensitivity. A clear link appears between the sensitivity and the image quality of the films in the low optical density range (mediastinal area). This relationship is less obvious in the high optical density range. This result shows that, at the present time, most of the manufacturers try to produce optimised screen-film systems. Thus, the optimisation task of chest imaging becomes less a matter of choosing a good system among bad systems, than a matter of finding a consensus among radiologists on the acceptable level of image quality.

\section{CONCLUSION}

The results show that most of the recent systems dedicated to chest imaging are optimised concerning dose and image quality, and this is especially true in the low optical density range where the quantum noise component is important. During this study it appeared that radiologists put a very strong weight on the resolution parameter when assessing image quality. Thus, the global figure of merit, which integrates contrast, noise and resolution, did not appear adquate to model the radiologists when assessing image quality.

The main problem which remains to be solved is the definition of the level of image quality which produces adequate images without including an important surplus of information. Since a clear link exists between dose and image quality, the definition of reference dose levels might be the way to set the level of image quality.

Table 4. Results of the objective assessment in the high-low optical density regions.

\begin{tabular}{|c|c|c|c|c|c|c|}
\hline System & Sensitivity & $\begin{array}{c}\text { High contrast } \\
\text { (OD) }\end{array}$ & $\begin{array}{c}\text { Low contrast } \\
\text { (OD) }\end{array}$ & Noise $\left(\mu \mathrm{m}^{2}\right)$ & $\begin{array}{l}\text { Resolution } \\
\text { Ip.mm }{ }^{-1} \text { at } \\
\text { MTF }=4 \%\end{array}$ & $\mathrm{GFM}(\times 1000)$ \\
\hline Kodak & 440 & $0.031-0.014$ & $0.0079-0.006$ & $72-36$ & 5.6 & $273-307$ \\
\hline Dupont & 450 & $0.046-0.024$ & $0.0089-0.004$ & $73-83$ & 5.3 & $339-274$ \\
\hline Fuji & 250 & $0.050-0.024$ & $0.0076-0.006$ & $123-37$ & 6.3 & $870-712$ \\
\hline Typon & 510 & $0.042-0.022$ & $0.0089-0.006$ & $65-76$ & 4.4 & $452-262$ \\
\hline Agfa & 230 & $0.032-0.018$ & $0.0087-0.004$ & $33-51$ & 5.8 & $451-293$ \\
\hline Imation & 280 & $0.032-0.014$ & $0.010-0.006$ & $26-30$ & 4.8 & $228-162$ \\
\hline
\end{tabular}

\section{REFERENCES}

1. ISO. Photography-Sensitometry of Screen-Film Systems for Medical Radiography - ISO Method Determination of Sensitometric Curve, Shape, Speed and Average Gradient. ISO CD 9236, Part 1 (1993).

2. Sicherung der Bildqualität in röntgendiagnostischen Betrieben. Abnahmeprüfungan an medizinischen Röntgen-Einrichtungen Funktionsprüfung der Filmverarbeitung. DIN 6868, Teil 55 (1991).

3. AAPM. Standardized Methods for Measuring Diagnostic X-Ray Exposure. AAPM Report 31 (1990).

4. Bildregistrierendes Systems Bestehend aus Röntgenfilms, Verstärkungsfolien und Kassetten zur Verwendung in der medizinischen Röntgendiagnostik. DIN 6867, Teil 1 (1985).

5. Coltman, J. W. The Specification of Imaging Properties by Response to a Sine Wave Input. J. Opt. Soc. Am. 44(6), 468471 (1954)

6. ICRU. Medical imaging. The assessment of image quality. Report 54 (Bethesda MD: ICRU Publications) (1996).

7. Bunch, P. C. Performance Characteristics of Asymmetric Zero-crossover Screen-Film Systems. SPIE 1653, 46-65 (1992). 